\title{
Journal of the Operations Research Society of China
}

\author{
Ya-xiang Yuan
}

Published online: 22 March 2013

(C) Operations Research Society of China, Periodicals Agency of Shanghai University, and

Springer-Verlag Berlin Heidelberg 2013

I am very pleased to present the first issue of Journal of the Operations Research Society of China. I believe that the launch of this new journal is not only a festival for the Operations Research Society of China, but also an important event in the international operational research community.

Economical globalization has dramatically changed the economical systems. Problems and difficulties crop up in optimization and decision making when we try to do dynamic reformations in energy, transportation, telecommunication, financial engineering, urban planning, health care, environmental pollution, natural resource consumption and transnational logistics. In solution of the complicated problems, traditional theories and methodologies of operations research and management science prove to be less useful. We have to find way out. We need to better understand the backgrounds and nature of the intrinsic system. We endeavor to develop new theories, methodologies and modeling approaches.

I hope, this new journal, launched by the Operations Research Society of China, will timely introduce problems in practical optimization and decision making, and report the advances in their solutions. I wish that Journal of the Operations Research Society of China be a global forum for the entire community of operations research and management science.

Y.-x. Yuan $(\bowtie)$

State Key Laboratory of Scientific/Engineering Computing, Institute of Comptational Mathematics and Scientific/Engineering Computing, AMSS, Chinese Academy of Sciences, Zhong Guan Cun Donglu 55, Beijing 100190, P.R. China

e-mail: yyx@1sec.cc.ac.cn 
I am happy and proud that we have a qualified international editorial board, that is sure to accomplish the mission of this journal. Of course, our goal cannot be achieved without the support from all optimizers and practitioners. The success of this journal depends on your care, support and contributions.

Editor-in-Chief 